\title{
Phase shift of cyclotron orbits at type-I and type-II multi-Weyl nodes
}

\author{
M. Breitkreiz, ${ }^{1,2}$ N. Bovenzi, ${ }^{1}$ and J. Tworzydło ${ }^{3}$ \\ ${ }^{1}$ Instituut-Lorentz, Universiteit Leiden, P.O. Box 9506, 2300 RA Leiden, The Netherlands \\ ${ }^{2}$ Dahlem Center for Complex Quantum Systems and Fachbereich Physik, Freie Universität Berlin, 14195 Berlin, Germany \\ ${ }^{3}$ Institute of Theoretical Physics, Faculty of Physics, University of Warsaw, ulitsa Pasteura 5, 02-093 Warszawa, Poland
}

(Received 3 July 2018; published 4 September 2018)

\begin{abstract}
Quantum oscillations of response functions in high magnetic fields tend to reveal some of the most interesting properties of metals. In particular, the oscillation phase shift is sensitive to topological band features, thereby helping to identify the presence of Weyl fermions. In this work, we predict a characteristic parameter dependence of the phase shift for Weyl fermions with tilted and overtilted dispersion (type-I and type-II Weyl fermions) and an arbitrary topological charge (multi-Weyl fermions). For type-II Weyl fermions our calculations capture the case of magnetic breakthrough between the electron and the hole part of the dispersion. Here, the phase shift turns out to depend only on the quantized topological charge due to the cancellation of nonuniversal contributions from the electron and the hole part.
\end{abstract}

DOI: 10.1103/PhysRevB.98.121403

Introduction. Electrons moving along cyclotron orbits in a homogeneous magnetic field are subject to the quantization condition [1]

$$
l^{2} S=2 \pi(m+\gamma), \quad m \in \mathbb{Z},
$$

where $S$ is the zero-field area enclosed by the cyclotron orbit in momentum space, $l=\sqrt{\hbar / e B}$ is the magnetic length, and the offset $\gamma$ includes quantum corrections, which can be expanded in powers of the magnetic field $B$ [2]. In the semiclassical regime when the magnetic length is much larger than the Fermi wavelength, field-dependent corrections to $\gamma$ are suppressed and the remaining number of zeroth order in $B$ encodes valuable information about the electronic properties of the system. In particular, the offset includes contributions coming from topological features in the band structure [3-6], which makes it the subject of high current interest. Experimentally, it can be deduced from quantum oscillations in the de Haasvan Alphen or the Shubnikov-de Haas effects, widely used nowadays to identify Weyl, Dirac, and nodal-line semimetals [7-11].

Interestingly, in some well-studied systems the offset measures the topological features independent of the specifics of the band structure. So, e.g., in graphene and graphene bilayer exposed to an out-of-plane magnetic field, the offset turns out to be given by a winding number-the number of full turns made by the direction of the electron's pseudospin degree of freedom during a single turn around the cyclotron orbit $[3,5]$. This integer winding number is a robust feature, determined by the type of the band touching, and is sometimes called the topological charge of the Weyl or Dirac fermion [12]. In contrast to the common belief, however, the topological charge contributes to the offset in such a robust manner only in exceptional cases, namely, when particular symmetry constraints are satisfied [6]. In general, the offset is sensitive also to other parameters of the band touching and it is the aim of this work to characterize this sensitivity.
One important parameter is a linear tilt of the dispersion at the Weyl node, which is generically present in material realizations and, most importantly, leads to the occurrence of two types of Weyl nodes, as sketched in Fig. 1. Upon the type-I to type-II transition, the tilt exceeds a critical value, above which an equienergy surface near the node cuts both bands [13]. The closed cyclotron orbit at a type-I Weyl node is thereby replaced by two open branches, which can be closed at large momenta by higher-order corrections to the Weyl Hamiltonian, resulting in two cyclotron orbits, one electronlike and one holelike. Band details determine a critical magnetic field, above which the two separate cyclotron orbits effectively merge into a single orbit via magnetic breakthrough $[14,15]$. This critical field is zero if the energy and the parallel momentum are exactly at the node where the two contours touch [16,17], and is larger than zero if the gap between the contours is finite. The magnetic breakdown contributes an additional phase to the offset $\gamma$, so one would expect that the offset is even more sensitive to details of the orbit than in the case without magnetic breakdown.

In this Rapid Communication, we analyze the offset for orbits at both types of Weyl nodes and find a characteristic

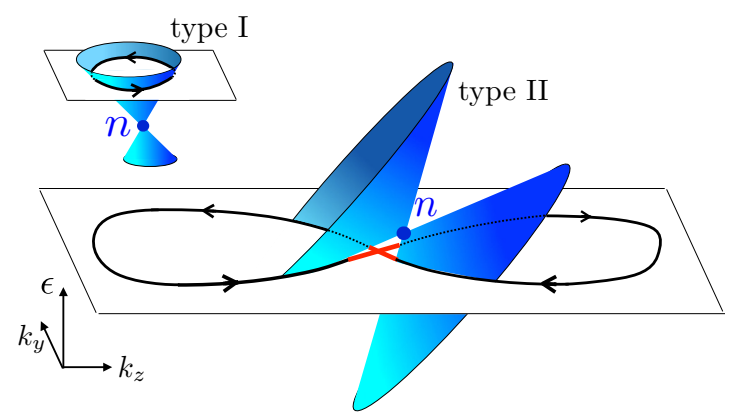

FIG. 1. Schematic illustration of a breakthrough cyclotron orbit (figure-8 curve) at a type-II Weyl node with topological charge $n$. The red part indicates quantum tunneling in the magnetic-breakthrough region. The inset shows a cyclotron orbit at a type-I Weyl node. 
dependence of $\gamma$ on the Weyl-node parameters. Most surprisingly, the offset of the breakthrough orbit at a type-II Weyl point turns out to depend only on the topological charge. This striking result is based on two facts, the universality of the phase jump of $\pi$ acquired in the magnetic-breakthrough region and a robust phase shift of $n \pi$ induced by the topological charge. The insensitivity of the latter on details of the orbit comes from a cancellation of a nonuniversal part of the phase in the two loops of the breakthrough orbit, which are traversed in opposite directions.

Model. We consider a set of Hamiltonians that govern the physics close to topologically distinct band touchings,

$$
\begin{aligned}
& H_{0}=k_{-} \sigma_{+}+k_{+} \sigma_{-}+u k_{z} \sigma_{0}, \\
& H_{n}=k_{-}^{n} \sigma_{+}+k_{+}^{n} \sigma_{-}+u k_{z} \sigma_{0}+k_{z} \sigma_{z}, \quad n \in\{1,2, \ldots\},
\end{aligned}
$$

where $k_{ \pm}=k_{x} \pm i k_{y},\left(k_{x}, k_{y}, k_{z}\right)=\mathbf{k}$ are momenta (scaled by velocities), $\sigma_{ \pm}=\sigma_{x} \pm i \sigma_{y}, \sigma_{x, y, z}$ are Pauli matrices, and $\sigma_{0}$ the identity matrix. The band touching at $\mathbf{k}=\mathbf{0}$ described by $H_{n}$ corresponds to a topologically protected multi-Weyl node of order $n$ [18], while $H_{0}$ describes a trivial, nonprotected band touching (a gap is produced by a perturbation $\propto \sigma_{z}$ ). The parameter $u>0$ controls the tilt of the Weyl cone; for $u<1$ and $u>1$ the Weyl cone is of type I and II, respectively.

The magnetic field pointing in the $x$ direction moves the particles along equienergy contours $k_{z}\left(k_{y}\right)$ at fixed energy $\epsilon$ and parallel momentum component $k_{x}$. The contours are determined by the Schrödinger equation

$$
H_{n}\left|u_{n \pm}\right\rangle=\epsilon\left|u_{n \pm}\right\rangle
$$

where \pm denote the two bands.

In the quantization condition (1) one can distinguish three phase shifts that contribute to the offset,

$$
\gamma=\frac{1}{2 \pi}\left(\phi_{0}+\phi_{\mathrm{b}}+\phi_{\mathrm{t}}\right)
$$

Here, $\phi_{0}$ and $\phi_{\mathrm{b}}$ are phase shifts that occur at singular points on the orbit. Specifically, turning points give rise to the Maslov phase $\phi_{0}$ [19], in which each turning point contributes a phase jump of $\pm \pi / 2$, the sign determined by the sign of the curvature at the turning point. In particular, one finds that $\phi_{0}=\pi$ and $\phi_{0}=0$ for orbits that can be deformed into a circle and into a figure- 8 shape, respectively. With $\phi_{\mathrm{b}}$ we denote the phase shifts that occur due to magnetic breakdown. Finally, $\phi_{\mathrm{t}}$ is the topological phase shift, which includes the Berry phase accumulated during a full turn around the orbit and the effect of the orbital magnetic moment $[4-6,20]$. The explicit calculation of $\phi_{\mathrm{b}}$ and $\phi_{\mathrm{t}}$ is the main result of this work, which will be presented in the following.

Topological phase shift. The topological phase shift of a closed contour at energy $\epsilon$ and the fixed momentum component $k_{x}$ is given by $[5,21]$

$$
\phi_{\mathrm{t}}=\oint d k_{y}^{\prime}\left[A-\frac{d k_{z}\left(k_{y}^{\prime}\right)}{d \epsilon} M\right] .
$$

Here, the first term is determined by the Berry connection projected onto the contour,

$$
A=i\left\langle u\left|\nabla_{\mathbf{k}}\right| u\right\rangle \cdot \frac{d \mathbf{k}}{d k_{y}}=i\left\langle u\left|\frac{d}{d k_{y}}\right| u\right\rangle,
$$

which contributes to $\phi_{\mathrm{t}}$ the usual Berry phase of the closed orbit. The second term is the correction to the zero-field area $S$ coming from the orbital magnetic moment projected onto the direction of the magnetic field [20],

$$
\begin{aligned}
M= & \frac{i}{2}\left[\left(\partial_{k_{y}}\langle u|\right)(\epsilon-H)\left(\partial_{k_{z}}|u\rangle\right)\right. \\
& \left.-\left(\partial_{k_{z}}\langle u|\right)(\epsilon-H)\left(\partial_{k_{y}}|u\rangle\right)\right] .
\end{aligned}
$$

The eigenfunctions of the Hamiltonian $H_{i}$ can be written as

$$
\begin{aligned}
\left|u_{0 \pm}\right\rangle & =\frac{1}{\sqrt{2}}\left(\begin{array}{c}
\mp e^{-i \alpha} \\
1
\end{array}\right), \\
\left|u_{n+}\right\rangle & =\left(\begin{array}{c}
-\sin \frac{\beta}{2} e^{-i n \alpha} \\
\cos \frac{\beta}{2}
\end{array}\right), \quad\left|u_{n-}\right\rangle=\left(\begin{array}{c}
\cos \frac{\beta}{2} e^{-i n \alpha} \\
\sin \frac{\beta}{2}
\end{array}\right),
\end{aligned}
$$

where the angles $\alpha$ and $\beta$ are defined as

$$
\begin{aligned}
\cos \beta & =\frac{k_{z}}{k}, \quad \sin \beta=\frac{\left(k_{x}^{2}+k_{y}^{2}\right)^{\frac{n}{2}}}{k}, \\
\alpha & =\arg \left(k_{x}+i k_{y}\right), \quad k=\sqrt{\left(k_{x}^{2}+k_{y}^{2}\right)^{n}+k_{z}^{2}} .
\end{aligned}
$$

For the topologically trivial case we obtain from (6)-(9)

$$
A_{0 \pm}=\frac{k_{x}}{2\left(k_{x}^{2}+k_{y}^{2}\right)}, \quad M_{0 \pm}=0,
$$

and the topological phase shift vanishes as it should,

$$
\phi_{\mathrm{t}}^{ \pm}=\oint d k_{y}^{\prime} A_{0 \pm}=\oint d k_{y}^{\prime} \frac{k_{x}}{2\left[k_{x}^{2}+\left(k_{y}^{\prime}\right)^{2}\right]}=0 \quad(n=0),
$$

independent of the integration contour. For the nontrivial case, we obtain

$$
A_{n \pm}=\frac{n k_{x}\left(k_{x}^{2}+k_{y}^{2}\right)^{n-1}}{2 k\left(k \pm k_{z}\right)}, \quad M_{n \pm}=-\frac{n k_{x}\left(k_{x}^{2}+k_{y}^{2}\right)^{n-1}}{2 k^{2}} .
$$

To calculate the topological phase shift, we consider the explicit expression for the equienergy contours, which is derived from (3) in the form

$$
k_{z}^{ \pm}\left(k_{y}\right)=\frac{\epsilon u \pm \sqrt{\left(u^{2}-1\right)\left(k_{x}^{2}+k_{y}^{2}\right)^{n}+\epsilon^{2}}}{u^{2}-1} .
$$

For $u>1$, the contours given by $k_{z}^{ \pm}\left(k_{y}\right)$ are disjoint and we need to introduce an additional orbit segment that connects the two open ends of $k_{z}^{ \pm}\left(k_{y}\right)$ at $k_{z} \rightarrow \pm \infty$. These connecting segments can be realized by an additional mass term $\eta k_{z}^{3} \sigma_{z}$ in the Hamiltonian, with an infinitesimal $\eta>0$. The reconnection then occurs at large momenta $k_{z}$, with $\left|k_{z}\right|>(u-1) / \eta \rightarrow \infty$. In the expressions (12) for $A$ and $M$ the additional mass term replaces $k_{z} \rightarrow k_{z}+\eta k_{z}^{3}$. On the connecting segment, $A$ and $M$ go to zero as $\eta^{2}$, while the integration along the connecting segment gives a factor of order $1 / \eta$. Hence the contribution of the connecting segment to $\phi_{\mathrm{t}}$ vanishes and the integration reduces to the integration along the main contour $k_{z}^{ \pm}\left(k_{y}\right)$. 
Inserting (12) and (13) into (5) we obtain

$$
\phi_{\mathrm{t}}^{ \pm}=\mp \int d k_{y}^{\prime} \frac{(u+1) n k_{x}\left[k_{x}^{2}+\left(k_{y}^{\prime}\right)^{2}\right]^{n-1}}{2\left[k^{ \pm} \pm k_{z}^{ \pm}\right]\left[k_{z}^{ \pm} \mp u k^{ \pm}\right]} .
$$

For a type-II cone $(u>1)$ we use the substitution $\kappa=k_{y}^{\prime} / k_{x}$ and obtain

$$
\begin{aligned}
\phi_{\mathrm{t}}^{ \pm}= & \int_{-\infty}^{\infty} d \kappa \frac{n\left(\kappa^{2}+1\right)^{n-1}}{2 \sqrt{\left(\kappa^{2}+1\right)^{n}+\cot ^{2} \theta}} \\
& \times\left(\sqrt{\left(\kappa^{2}+1\right)^{n}+\cot ^{2} \theta} \pm \cot \theta\right)^{-1}
\end{aligned}
$$

where the parameter $\theta$ encoding contour details is defined as

$$
\theta= \begin{cases}\arctan \left(\frac{k_{x}^{n} \sqrt{u^{2}-1}}{\epsilon}\right), & u>1, \\ \operatorname{arctanh}\left(\frac{k_{x}^{n} \sqrt{1-u^{2}}}{\epsilon}\right), & u<1 .\end{cases}
$$

The integral in (15) needs to be calculated numerically (see below); for the special case $n=1$, we find the closed-form solution

$$
\phi_{\mathrm{t}}^{ \pm}=\frac{\pi}{2}(1 \mp \operatorname{sgn} \theta) \pm \theta \quad(n=1) .
$$

While $\phi_{\mathrm{t}}^{ \pm}$are the topological phase shifts of the two (electron/hole) orbits $k_{z}^{ \pm}\left(k_{y}\right)$, the sum $\phi_{\mathrm{t}}^{+}+\phi_{\mathrm{t}}^{-} \equiv \phi_{\mathrm{t}}^{\mathrm{br}}$ is the topological phase shift of the breakthrough orbit, i.e., the figure- 8 orbit that encloses both the electron and the hole pocket. Using the substitution $z=\left(\kappa^{2}+1\right)^{n}$, the integral for $\phi_{\mathrm{t}}^{\mathrm{br}}$ simplifies to

$$
\phi_{\mathrm{t}}^{\mathrm{br}}=\int_{1}^{\infty} d z \frac{1}{\sqrt{z^{\frac{2 n+1}{n}}-z^{2}}}=n \pi,
$$

where the $\theta$-dependent part cancels out. As a result, the topological phase shift of the figure- 8 orbit only depends on the quantized topological charge $n$, in contrast to the $\theta$-dependent phase shifts of the separate orbits.

For type-I Weyl fermions $(u<1) k_{z}^{ \pm}$are two parts of a single closed contour, whose topological phase is denoted $\phi_{\mathrm{t}}$. A closed-form solution for the integral (14) is found for $n=1$,

$$
\phi_{\mathrm{t}}=\pi \operatorname{sgn} \theta \quad(n=1),
$$

in agreement with Refs. [3,5,22]. For $n \geqslant 2$, we find in the limits $\theta \rightarrow 0^{ \pm}$and $\theta \rightarrow \pm \infty[23]$

$$
\begin{aligned}
& \phi_{\mathrm{t}}=\stackrel{\theta \rightarrow 0^{ \pm}}{\longrightarrow} n \pi \operatorname{sgn} \theta, \\
& \phi_{\mathrm{t}}=\stackrel{\theta \rightarrow \pm \infty}{\longrightarrow} \sqrt{n} \pi \operatorname{sgn} \theta .
\end{aligned}
$$

The full $\theta$ dependence will be discussed below.

Breakthrough phase shift. To calculate the additional phase shift of the figure-8 orbit due to magnetic breakdown, we follow a standard route [15] and calculate the scattering matrix that relates the exact wave function of the magnetic-breakdown region with the in- and outgoing semiclassical wave functions.

We start with the nontopological Hamiltonian $H_{0}$. Introducing the magnetic field via Peierls substitution $k_{z} \mapsto k_{z}+$ $i l^{-2} \partial_{k_{y}}$, followed by a unitary transformation,

$$
\tilde{H}_{0}=e^{-i l^{2}\left(k_{z}-\epsilon / u\right) k_{y}} H_{0} e^{i l^{2}\left(k_{z}-\epsilon / u\right) k_{y}},
$$

we arrive at

$$
\tilde{H}_{0}=k_{x} \sigma_{x}+k_{y} \sigma_{y}+i u l^{-2} \partial_{k_{y}} \sigma_{0}+\epsilon .
$$

Rescaling the variables as $k=l k_{y} / \sqrt{u}, \delta_{0}=l k_{x} / \sqrt{u}$, the Schrödinger equation $\tilde{H}_{0} \psi=\epsilon \psi$ reads

$$
\left[\sigma_{x} \delta_{0}+\sigma_{y} k+i \partial_{k}\right] \psi=0
$$

The exact solution of (23) is known from the Landau-Zener problem [24]. To obtain the phase shift in comparison to the semiclassical solution of (23), the exact wave functions are matched with the incoming semiclassical wave functions at $k \ll-\delta_{0}$, denoted $\psi_{i}^{ \pm}$, and outgoing $\psi_{f}^{ \pm}$at $k \gg \delta_{0}$. From this standard procedure (recapitulated in the Supplemental Material [23]) we obtain the scattering matrix $S$ that relates the final state in the basis $\left(\psi_{f}^{+}, \psi_{f}^{-}\right)$to the incoming state in the basis $\left(\psi_{i}^{+}, \psi_{i}^{-}\right)$,

$$
S=\left(\begin{array}{cc}
\sqrt{1-W} e^{i \alpha} & -i \sqrt{W} \\
-i \sqrt{W} & \sqrt{1-W} e^{-i \alpha}
\end{array}\right)
$$

where

$$
W=e^{-\pi \delta_{0}^{2}}, \quad \alpha=\frac{\pi}{4}+\frac{\delta_{0}^{2}}{2}-\frac{\delta_{0}^{2}}{2} \ln \frac{\delta_{0}^{2}}{2}+\arg \Gamma\left(i \frac{\delta_{0}^{2}}{2}\right) .
$$

The breakthrough orbit dominates if $\delta_{0} \ll 1, W \approx 1$, in which case each band transition in the breakthrough region contributes a phase jump of $\pi / 2$ giving in total the phase shift $\phi_{\mathrm{b}}=\pi$ for the breakthrough orbit.

For the topological case, we linearize the Hamiltonian $H_{n}$ in $k_{y}$, leading to

$$
H_{n}^{\prime}=k_{x}^{n} \sigma_{x}+n k_{x}^{n-1} k_{y} \sigma_{y}+k_{z} \sigma_{z}+u k_{z} .
$$

After Peierls substitution we apply the unitary transformation given by

$$
\tilde{H}_{n}=e^{-i l^{2}\left[k_{z}-\epsilon /\left(u^{2}-1\right)\right] k_{y}} H_{n}^{\prime} e^{i l^{2}\left[k_{z}-\epsilon /\left(u^{2}-1\right)\right] k_{y}} .
$$

Rescaling and transforming the variables as

$$
\begin{aligned}
k & =l k_{y}\left(u^{2}-1\right)^{-1 / 4} \sqrt{n k_{x}^{n-1}}, \\
\delta_{n} & =l \operatorname{sgn}(\epsilon) \frac{\sqrt{\epsilon^{2}+\left(u^{2}-1\right) k_{x}^{2 n}}}{\left(u^{2}-1\right)^{3 / 4} k_{x}^{(n-1) / 2}},
\end{aligned}
$$

we obtain the Schrödinger equation

$$
\begin{aligned}
& {\left[\delta_{n} \sqrt{u^{2}-1} \sin \theta \sigma_{x}+k \sqrt{u^{2}-1} \sigma_{y}\right.} \\
& \left.\quad+i \partial_{k}\left(u+\sigma_{z}\right)+\delta_{n} \cos \theta\left(1+u \sigma_{z}\right)\right] \psi=0 .
\end{aligned}
$$

Multiplying (29) from the left with $\mathcal{M}=\operatorname{diag}\left[(u+1)^{-1},(u-\right.$ $\left.1)^{-1}\right]$ and applying a transformation given by

$$
T=-i\left(\begin{array}{cc}
\frac{1-u}{\sqrt{u^{2}-1}} & \frac{u-1}{\sqrt{u^{2}-1}} \\
1 & 1
\end{array}\right) \sigma_{z} e^{-i \sigma_{y} \theta / 2},
$$

we again arrive at the differential equation of the Landau-Zener form (23),

$$
\hat{H} \hat{\psi}(k)=\left(\delta_{n} \sigma_{x}+k \sigma_{y}+i \partial_{k}\right) \hat{\psi}(k)=0,
$$

where $\hat{\psi}(k)=T^{-1} \psi(k)$ and $\hat{H}=T^{-1} \mathcal{M} \tilde{H} T$. The solution of (29) is thus given by the solution of the Landau-Zener problem multiplied from the left with the matrix $T$. Note that the $\theta$ phase brought into the full solution by the matrix $T$ is the 


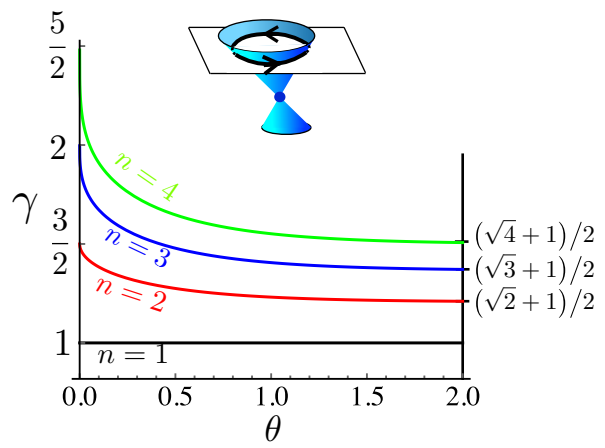

FIG. 2. Parameter dependence of the offset $\gamma$ of orbits at a type-I Weyl node.

topological phase of the full solution induced by the nontrivial topology of the Hamiltonian.

The $S$ matrix is obtained by matching $\psi(k)$ with the semiclassical solution of (29). Since $H_{n}^{\prime}$ is topologically equivalent to $H_{1}$ (note that the dynamical variables are $k_{y}$ and $k_{z}$, while $k_{x}$ is fixed), the topological phase shift of the semiclassical solution is given by (17), which cancels the $\theta$ phase of the full solution and the result is the same $\theta$-independent scattering matrix (24), with $\delta_{0}$ replaced by $\delta_{n}$. In particular, the breakthrough phase shift $\phi_{\mathrm{b}}=\pi$ also holds in the topological case.

Discussion. Having thus calculated the phase shifts, we now show the full $\theta$ dependence of the offset $\gamma$, defined in Eq. (4), in Figs. 2 and 3 [25]. For the figure- 8 orbit, the magnetic breakthrough contributes an offset $1 / 2$ and the topological charge adds an extra contribution $n / 2$. The $\theta$ independence is based on the cancellation of the $\theta$-dependent parts from the hole and the electron pockets. The universality of the breakthrough phase shift is, instead, less surprising, since the same universal value was found previously for nontopological band touchings [15].

In contrast, without breakthrough (dashed/dotted curves in Fig. 3) or in case of a type-I Weyl node (Fig. 2), the offset has a nontrivial dependence on the orbit details that are encoded in $\theta$. The only exception is the case $n=1$ of the type-I Weyl node, which shows no $\theta$ dependence owing to the higher symmetry of the dispersion [6]. This is also the only case with a known full quantum-mechanical solution [3,5,22]; it agrees with our semiclassical result. In quantum oscillation experiments, the measured phase shift would likely be averaged over a range of values of the energy and of the parallel momentum $k_{x}$, corresponding to a weighted (depending on details of the

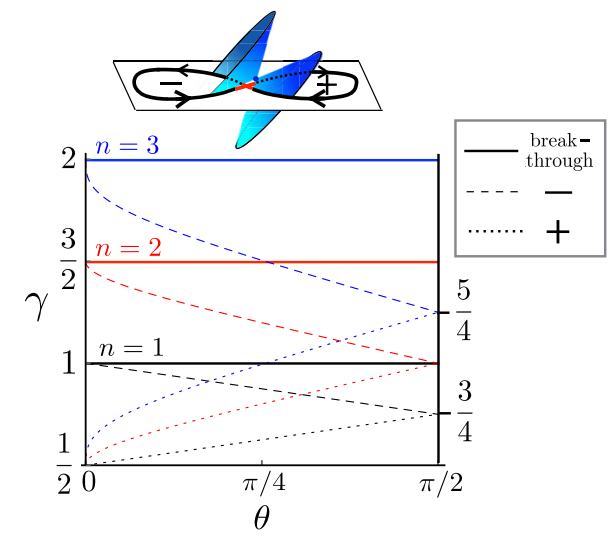

FIG. 3. Parameter dependence of the offset $\gamma$ of orbits at a type-II Weyl node. The offsets of separate orbits $k_{z}^{+}$and $k_{z}^{-}$(without magnetic breakthrough) depend on the band parameter $\theta$, while the offset of the figure- 8 breakthrough orbit only depends on the topological charge $n$.

experimental realization) average over the parameter $\theta$. In general, this averaging does not destroy the $\theta$ dependence, still allowing to discriminate the two cases of quantized and continuously varying $\gamma$.

With regard to the figure- 8 breakthrough orbits, our calculations explain recent numerical findings for the offset of a thin-film Weyl semimetal [26] and a type-II Weyl semimetal [16], showing, respectively, $\gamma=1 / 2$ and $\gamma=0$. In the case of the thin film, the Hamiltonian at the figure- 8 crossing, given in the Appendix of Ref. [26], is equivalent to the nontopological Hamiltonian $H_{0}$, thus the only phase contributing is the breakthrough phase $\phi_{\mathrm{b}}=\pi$, which explains the offset $\gamma=\phi_{\mathrm{b}} / 2 \pi=$ $1 / 2$. In case of the type-II Weyl semimetal, the Hamiltonian is equivalent to $H_{1}$, where the additional topological phase $\phi_{\mathrm{t}}=$ $\pi$ cancels the breakthrough phase, which explains the vanishing offset. This contradicts a previous interpretation that relates the vanishing offset of the latter to a vanishing Berry phase and neglects the contribution of the breakthrough phase [17]. In the Supplemental Material [23] we present extensions of the numerical calculations to the cases $n=2$ and $n=3$, tilted type-I Weyl cones, and several values of $\theta$. Also these calculations are in agreement with the analytical results of this work.

Acknowledgments. The authors would like to thank C. W. J. Beenakker, A. Alexandradinata, and M. J. Pacholski for valuable discussions. This research was supported by the Netherlands Organization for Scientific Research (NWO/OCW) and an ERC Synergy Grant.
[1] L. M. Roth, Phys. Rev. 145, 434 (1966).

[2] Y. Gao and Q. Niu, Proc. Natl. Acad. Sci. USA 114, 7295 (2017).

[3] G. P. Mikitik and Y. V. Sharlai, J. Exp. Theor. Phys. 87, 747 (1998).

[4] G. P. Mikitik and Y. V. Sharlai, Phys. Rev. Lett. 82, 2147 (1999).

[5] J. Fuchs, F. Piéchon, M. Goerbig, and G. Montambaux, Eur. Phys. J. B 77, 351 (2010).

[6] A. Alexandradinata, C. Wang, W. Duan, and L. Glazman, Phys. Rev. X 8, 011027 (2018).
[7] Y. Zhang, Y.-W. Tan, H. L. Stormer, and P. Kim, Nature (London) 438, 201 (2005).

[8] L. P. He, X. C. Hong, J. K. Dong, J. Pan, Z. Zhang, J. Zhang, and S. Y. Li, Phys. Rev. Lett. 113, 246402 (2014).

[9] X. Huang, L. Zhao, Y. Long, P. Wang, D. Chen, Z. Yang, H. Liang, M. Xue, H. Weng, Z. Fang, X. Dai, and G. Chen, Phys. Rev. X 5, 031023 (2015).

[10] J. Hu, Z. Tang, J. Liu, X. Liu, Y. Zhu, D. Graf, K. Myhro, S. Tran, C. N. Lau, J. Wei, and Z. Mao, Phys. Rev. Lett. 117, 016602 (2016). 
[11] S. Pezzini, M. R. van Delft, L. Schoop, B. Lotsch, A. Carrington, M. I. Katsnelson, N. E. Hussey, and S. Wiedmann, Nat. Phys. 14, 178 (2017).

[12] A. Burkov, Nat. Mater. 15, 1145 (2016).

[13] A. A. Soluyanov, D. Gresch, Z. Wang, Q. Wu, M. Troyer, X. Dai, and B. A. Bernevig, Nature (London) 527, 495 (2015).

[14] M. H. Cohen and L. M. Falicov, Phys. Rev. Lett. 7, 231 (1961).

[15] M. I. Kaganov and A. A. Slutskin, Phys. Rep. 98, 189 (1983).

[16] T. E. O'Brien, M. Diez, and C. W. J. Beenakker, Phys. Rev. Lett. 116, 236401 (2016).

[17] A. Alexandradinata and L. I. Glazman, Phys. Rev. Lett. 119, 256601 (2017).

[18] C. Fang, M. J. Gilbert, X. Dai, and B. A. Bernevig, Phys. Rev. Lett. 108, 266802 (2012).

[19] J. B. Keller, Ann. Phys. (NY) 4, 180 (1958).

[20] G. Panati, H. Spohn, and S. Teufel, Commun. Math. Phys. 242, 547 (2003).
[21] D. Xiao, M.-C. Chang, and Q. Niu, Rev. Mod. Phys. 82, 1959 (2010).

[22] Z.-M. Yu, Y. Yao, and S. A. Yang, Phys. Rev. Lett. 117, 077202 (2016); S. Tchoumakov, M. Civelli, and M. O. Goerbig, ibid. 117, 086402 (2016); M. Udagawa and E. J. Bergholtz, ibid. 117, 086401 (2016); G. P. Mikitik and Y. V. Sharlai, Phys. Rev. B 94, 195123 (2016).

[23] See Supplemental Material at http://link.aps.org/supplemental/ 10.1103/PhysRevB.98.121403 for details of analytical calculations andadditional numerical calculations supporting the analytical results.

[24] L. D. Landau and E. M. Lifshitz, Course of Theoretical Physics (Elsevier, Oxford, UK, 1977).

[25] In practice, the offset can only be measured modulo one, corresponding to one Landau-level spacing. Nevertheless, in Figs. 2 and 3 we plot the full $\gamma$ for clarity of the graphic.

[26] N. Bovenzi, M. Breitkreiz, T. E. O’Brien, J. Tworzydło, and C. W. J. Beenakker, New J. Phys. 20, 023023 (2018). 